\title{
A randomized controlled trial comparing self-referral to inpatient treatment and treatment as usual in patients with severe mental disorders
}

Running title: Self-referral as inpatients in mental disorders

Vidir Sigrunarson, MD ${ }^{1,2}$, Inger Elise Opheim Moljord MSc ${ }^{1,2}$, Aslak Steinsbekk, $\mathrm{PhD}^{3}$, Lasse Eriksen, $\mathrm{PhD}^{1,2}$, Gunnar Morken, $\mathrm{MD} \mathrm{PhD}^{2,4}$

${ }^{1}$ Nidaros Community Mental Health Centre, Division of Psychiatry, St. Olavs University Hospital, Trondheim, Norway

${ }^{2}$ Department of Neuroscience; Faculty of Medicine, Norwegian University of Science and Technology, Trondheim, Norway

${ }^{3}$ Department of Public Health and General Practice, Norwegian University of Science and Technology, Trondheim, Norway

${ }^{4}$ Department of Psychiatry, St Olav University Hospital, Trondheim, Norway

Corresponding author: Gunnar Morken, Department of Neuroscience; Faculty of Medicine, Norwegian University of Science and Technology, Trondheim, Norway

Address: Østmarka Department, St Olavs University Hospital, Box 3250, Sluppen, 7006

Trondheim, Norway

Telephone: +4772823034

Email: gunnar.morken@ntnu.no 


\section{Abstract}

Background: There has been a call for increased patient autonomy and participation in psychiatry. Some Community Mental Health Centers (CMHC) have implemented services called "self-referral to inpatient treatment" (SRIT) for patients with severe mental disorders.

Aims: To investigate whether SRIT could yield better outcomes after 12 months in use of mental health services for people with severe mental disorders than Treatment As Usual (TAU).

Methods: This was a randomized controlled trial at a CMHC in Norway comparing SRIT and TAU in 12 months. 54 patients with severe mental disorders were included. The patients in the SRIT group could admit themselves as inpatients for up to 5 days for each admission with at least a 2 weeks pause between the admittances.

Results: Twenty out of 26 participants (77\%) in the SRIT group used the SRIT for a median of 1.5 admissions and 5 inpatient days. With the exception of a somewhat larger number of admissions at the CMHC in the SRIT group, no significant differences were found between the 2 groups in days as inpatients, admissions, outpatient contacts or coercion. Both groups reduced their inpatients days with $40 \%$.

Conclusions: Both the SRIT and the TAU groups reduced their use of services during the 12 months intervention period. Giving patients with severe mental disorders the possibility to self-refer did not change the use of services.

Clinical implications: Self-referral to inpatient treatment for patients with severe mental disorders might increase patient autonomy, but do not seem to save use of inpatient services.

\section{Key words:}

Psychotic disorders, Admissions, Self-referral, Rehabilitation, Recovery 


\section{Background}

Models for increased patient autonomy and participation in treatment decisions (1) have been implemented both in psychiatry and other medical disciplines throughout the western world (2) and their effects documented (3). Increased engagement, knowledge and realistic perception of outcomes and higher rating of satisfaction are described both in more acute choices of treatment of somatic conditions and in chronic disorders as diabetes. Less progress has been made in patient autonomy and participation in treatment of patients with chronic psychiatric disorders, especially those with psychotic disorders (4). Schizophrenia and bipolar disorder (BD) are among the most costly illnesses in the world and account for a considerable share of total health care budgets in developed countries (5-7). A large proportion of the total health care costs of schizophrenia and BD are due to hospitalizations $(7,8)$. Rehospitalizations are costly and are markers of reduced function and quality of life of patients with psychotic disorders and their families (9-11). Internationally the concept of recovery has had a growing influence on mental health services (12) emphasizing patients self-direction, empowerment and responsibility (13). One solution to increase the patient participation in mental health services is to make it possible for patients to self-refer to inpatient stay in mental health services. Several Community Mental Health Centers (CMHC) in Norway have implemented services called "self-referral to inpatient treatment" (SRIT) for patients with severe mental disorders (14-16). The aims of SRIT are to secure low threshold access to short term inpatient admissions and to increase patient participation and involvement in treatment choices and decisions (17). There are no RCTs on the effects of SRIT, but one uncontrolled study comparing patients before and after inclusion in SRIT, found that over a period of approximately 18 months, the number of admissions rose, but the number of days hospitalized fell by $1 / 3$ and hospitalization days by coercion were approximately halved (14). 


\section{Aims}

The aim of this randomized controlled trial was to investigate whether having a contract to use SRIT had an effect on use of mental health services, including use of hospital beds, over 12 months for people with severe mental disorders compared with treatment as usual (TAU) which both groups received.

Our primary hypothesis was that the total number of inpatient days in mental health services would be significantly fewer for patients randomized to SRIT compared with TAU over a 12 month period.

Secondary hypotheses were that use of admissions and inpatient days at CMHC, psychiatric hospital acute wards and use of coercion would be significantly fewer in the SRIT group compared with the TAU group as assessed over a 12 month period.

\section{Materials and methods}

\section{Trial design}

This was an open parallel group randomized controlled trial, with a balanced randomization between the SRIT and the TAU group. Written informed consent was obtained prior to randomization and the research was carried out in compliance with the Helsinki Declaration. The study was approved both by The Regional Committee for Medical and Health Research Ethics in Central-Norway (No 2009/1704) and the Norwegian Data Inspectorate and registered in Clinicaltrials.gov (NCT 01133587). The inclusion period was between May 2010 and December 2012.

\section{Study settings}

The study took place at the Rehabilitation section of Nidaros CMHC in Trondheim, Norway. The CMHC has a catchment area with a total of 94000 inhabitants, two-thirds urban and the rest from surrounding rural areas. The CMHC offer both in- and outpatient services including 
home visits and group activities. Nidaros CMHC is a part of St Olavs University Hospital which is the only provider of psychiatric CMHC and hospital inpatient services in the area.

\section{Participants}

All participants were extensive users of services over at least two years both from primary and psychiatric health care and had prior admittance to the rehabilitation section of the trial CMHC. Examples of received services were regular or long time admissions, frequent supervision from psychiatric nurses, outpatient contacts, and supportive housing residency, for details, see Results.

Patients with severe self-destructive or unrestrained behavior, ongoing drug or alcohol abuse or who did not acknowledge the need for or the function of SRIT were excluded. Patients with known previous alcohol or drug abuse were not excluded.

The ICD-10 clinical diagnoses (18) were set according to the routines of the department either by a psychiatrist or a senior clinical psychologist before the participants were included in the study.

All participants, except two, were diagnosed with bipolar disorders or schizophrenia, one participant was diagnosed with an organic psychosis and one with a personality disorder with psychotic features (voice hallucinations).

\section{Recruitment}

Most of the participants were recruited following a stay in the rehabilitation section of the CMHC offering SRIT, some were included and randomized while still admitted at the CMHC, expected to be discharged in a short time. Written and oral information were given to the patients in an information meeting about the study, with an invitation to participate. The participants were then contacted through their therapists or other health care personnel at the 
CMHC. Patients and their relatives could also take direct contact for information and enrollment in the program. All participants gave their written consent. All participants had to be approved by the chief physician.

\section{Interventions}

Participants in the intervention group signed a one year contract on "self-referral to inpatient treatment" (SRIT). The participants assigned to the SRIT intervention could, at their own initiative, admit themselves to the rehabilitation section as inpatients for up to 5 days for each admission without referral from a physician or other health care personnel in advance, with a mandatory pause of 14 days between admissions. Participants could self-refer to the rehabilitation section of the CMHC on Mondays to Fridays between 08:00 and 20:00. This was done to avoid capacity problems in the unit and to follow the routines from the first study in Norway (14). During the stay, the patients participated in the daily routines of the section, but did not receive any specific treatment beyond that as part of the stay, i.e. consultations about medications or other mental health related problems.

Patients in the control group received treatment as usual (TAU) and were offered a contract on SRIT one year after inclusion in the study. All participants, regardless of group allocation, had the opportunity to be admitted through regular procedures. TAU consisted of ordinary psychosocial and pharmacological treatment including contact with the general practitioner, emergency department, or duty doctor if they needed hospitalization. All participants were motivated to establish an individual treatment plan describing services they received and what to do if symptoms increased in severity, which almost all patients had when they joined the study.

\section{Outcomes}

The primary outcome was inpatient days in any psychiatric department at the university hospital or CMHC from baseline to 12 months after inclusion. Secondary endpoints were 
inpatient days and admissions at the CMHC, both included and excluded SRIT, hospital acute and rehabilitation wards and use of coercion. To document the implementation of the intervention, number of SRIT admissions, and days admitted were recorded. The data were collected from the patient administrative systems of the hospital trust.

\section{Sample size}

Sample size was not calculated based on the primary outcome, days as inpatients, but based on estimated number of patients that it would be possible to include during the two and a half year inclusion period.

\section{Randomization}

An internet based computerized randomization service from Norwegian University of Science and Technology (NTNU) was used for the concealed sequence generation. Stratification was done: Patients were stratified in those who had Psychiatric Ambulatory Rehabilitation Team or not. Block randomization was used and all involved in the study were blinded to the size of the blocks.

\section{Blinding}

There were no blinding of results in the study; the results are based on patient administrative data.

\section{Statistical methods}

Data were analyzed according to the intention to treat principle. Parametric tests were used to analyze normally distributed continuous data and nonparametric tests to analyze not normally distributed data. Categorical data were analyzed using Chi-Square test. ANCOVA was used to control for possible effects of covariates. For the within group analysis, paired t-test was used. The alpha level was set to .05. The statistical analyzes were done with SPSS version 21 . 


\section{Results}

\section{Participant flow}

The number of participants assessed for eligibility was not available. 54 participants meeting the inclusion criteria were randomized from May 2010 to December 2012. Of the 54 recruited, one participant withdrew from the project immediately following randomization to the control group and was not included in the analysis. Of the remaining 53, one participant in the intervention group died 9 months and 9 days after inclusion and one in the control group died just short of 6 months after inclusion. Data on deceased participants were included in the analysis on an intention to treat basis. See Figure 1.

$<($ Figure 1$)>>$

\section{Baseline data}

Baseline demographics and clinical characteristics were similar in both groups apart from participants in the intervention group being older. The participants in the intervention group had non-significantly more severe scores on all clinical parameters, except for diagnoses, see Table 1.

$<<($ Table 1$)>>$

\section{Implementation of the intervention}

Twenty of 26 participants (77\%) in the SRIT group used the opportunity of self-referral and had a median of 1.5 admissions and 5 inpatient days during the 12 months SRIT intervention.

\section{Outcome}

The median number of days as inpatients during 12 months were 39.5 (mean 81.4) days in the SRIT group and 33 (mean 66.6) days in the TAU group with a mean difference of 14.7 days (Standard Error 23.9 and 95\% Confidence Interval -33.2 to 62.7 ) and $\mathrm{p}=.54$.

For the secondary outcomes, with the exception of larger number of admissions at CMHC in the SRIT group, no significant differences were found between the groups, see Table 2. 
$<($ Table 2$)>>$

Analyses of covariance (ANCOVA) controlling for: age $(\mathrm{F}=.08, \mathrm{p}=.78)$, days hospitalised 12 months before inclusion $(\mathrm{F}=.35, \mathrm{p}=.56)$ and inpatient days under coercion the preceding year $(\mathrm{F}=.29, \mathrm{p}=.60)$ showed no significant differences between the intervention and control groups in days hospitalised in the 12 months after inclusion.

\section{Ancillary analyses}

A paired t-test for within group changes showed that both groups as a whole had a significant reduction in the total number of inpatient days 12 months after randomization compared to 12 months before randomization $($ mean $=56.6$ days, $\mathrm{SD}=145.2,95 \% \mathrm{CI}=16.6-96.6, \mathrm{p}=$ 0.006). The mean reduction for the intervention group was 63.3 days (140.8 days before and 77.5 days after), $\mathrm{SD}=156.6,95 \% \mathrm{CI}=0.05-126.6, \mathrm{p}=.05$ and the mean reduction for the control group was 50.1 days (113.4 days before and 63.3 days after), $\mathrm{SD}=136.1,95 \% \mathrm{CI}=-$ $3.7-103.9, \mathrm{p}=.067$.

\section{Discussion}

\section{Synopsis of the key findings}

We found no significant differences in use of psychiatric services between the intervention group and the TAU group except for more admissions to the $\mathrm{CMCH}$ in the SRIT group. Paired t-tests showed a significant reduction (approximately 40\%) in the number of days admitted 12 months after randomization compared to 12 months before randomization both in the intervention group and the control group. To our knowledge, this is the first randomized controlled trial on the effects of self-referral to inpatient treatment for patients with severe mental disorders. 


\section{Consideration of possible mechanisms and explanations}

Participants in the intervention group were considerably older than in the control group. Age is known to affect prognosis and the extent of use of psychiatric services of patients with psychotic disorders (19). An ANCOVA test controlling for age showed however no differences between the intervention group and the control group in number of days hospitalized 12 months after inclusion.

All the participants had good access to services, severe illnesses and low function described by their diagnoses and the number of days as inpatients the year before inclusion in the study. This might explain the lack of effects of a rather limited intervention as SRIT compared to the TAU intervention that all the participants in both groups received.

The present study focused on a limited scope of objective outcomes, and possible effects of SRIT on other aspects of recovery were not addressed. (20) (21) (22) Some of these themes were analyzed in a qualitative study, based on the same material where a selection of the patients was interviewed 4 and 12 months after inclusion in the study $(15,23)$.

Due to outliers in use of inpatient days and admissions there is a great difference between the median and mean values.

A possible explanation of the reduction in use of services in both groups might be that patients were referred to the trial during a period of exacerbation in their illness, and therefore had a higher level of use of services than usual. Thus a normalization of the use of services after inclusion may explain some of the reduction during the 12 months intervention period in addition to regression to the mean seen in many studies. Another explanation might be the effects of increased contact and assessments of patients in both groups during the intervention period. A third explanation might be that the patients in the TAU group knew that they would have a contract on SRIT one year later. 


\section{Comparison with relevant findings from other published studies.}

No other RCT's are found on the effects of SRIT. One uncontrolled study observing patients before and after they were included in SRIT found that over a period of approximately 18 months, the number of admissions rose, but the number of day's hospitalized and hospitalization days by coercion were reduced (14). These results are comparable to our findings from within group analyzes showing a reduction in use of services in both the intervention and the control groups of $40 \%$ over 12 months.

\section{Limitations and strengths of the present study}

A limitation of the study is that no prior power calculation could be made based on the main outcome as previous data were not available. A sample size of 53 participants might be too small to reveal minor differences between the two treatment groups, but major differences should be revealed. The cost and resource utilization of the intervention was not compared with the standard cost of CMHC-admissions. The patients were not systematically diagnosed at inclusion with a diagnostic tool as SCID-I (24), but on clinically based ICD-10 diagnoses which had been confirmed over time as the patients had received treatment both as in- and out-patients several times before entering the study both at the hospital and CMHC department with diagnostic evaluations. The present study measured one objective dimension of outcome i.e. use of services while other possible effects of SRIT remain to be evaluated. It might take up to several years to see marked improvement in symptoms in schizophrenia (25), therefore a follow up period of 12 months might be short to see long term clinical improvements resulting in lesser use of psychiatric services (26). Strengths of the study are the randomized controlled design and the objective data on use of services on all patients from psychiatric health care services. 


\section{Clinical and research implications of the work.}

Giving patients with severe mental disorders the possibility to self-refer did not change the use of services during the 12 months intervention period compared to TAU. Thus SRIT admissions seemed to replace some of the ordinary admissions and give some more autonomy to the patients. Further research is needed on both short- and long term effects of SRIT focusing on multiple aspects of recovery before it is possible to conclude about the clinical value of this treatment procedure.

\section{Acknowledgements}

We are extremely grateful to all the patients and their families who took part in this study, the staff for their help in recruitment and treatment in the study, and interviewers and clerical workers.

\section{Funding}

The study was financed by St Olavs University Hospital, Trondheim, Norway

\section{Disclosure of interests.}

The authors declare that they have no competing interests. 


\section{References}

1. Goldman HH. Mental health: A report of the Surgeon General: Ohio Department of Mental Health; 2000.

2. Elwyn G, Frosch D, Thomson R, Joseph-Williams N, Lloyd A, Kinnersley P, et al. Shared decision making: a model for clinical practice. J Gen Intern Med. 2012;27(10):1361-7.

3. Stacey D, Bennett CL, Barry MJ, Col NF, Eden KB, Holmes-Rovner M, et al. Decision aids for people facing health treatment or screening decisions. Cochrane Database Syst Rev. 2011;10(10).

4. Hamann J, Leucht S, Kissling W. Shared decision making in psychiatry. Acta Psychiatr Scand. 2003;107(6):403-9.

5. Knapp M, Mangalore R, Simon J. The global costs of schizophrenia. Schizophr Bull . 2004;30(2):279.

6. Thornicroft G, Tansella M, Becker T, Knapp M, Leese M, Schene A, et al. The personal impact of schizophrenia in Europe. Schizophr Res. 2004;69(2):125-32.

7. Kleine-Budde K, Touil E, Moock J, Bramesfeld A, Kawohl W, Rössler W. Cost of illness for bipolar disorder: a systematic review of the economic burden. Bipolar Disord. 2014;16(4):337-53. 8. Martin BC, Miller LS. Expenditures for treating schizophrenia: A population-based study of Georgia Medicaid recipients. Schizophr Bull. 1998;24(3):479.

9. Almond S, Knapp M, Francois C, Toumi M, Brugha T. Relapse in schizophrenia: costs, clinical outcomes and quality of life. Br J Psychiatry. 2004;184(4):346-51.

10. Weiden PJ, Olfson M. Cost of relapse in schizophrenia. Schizophr Bull. 1995;21(3):419-29. 11. Ascher-Svanum H, Zhu B, Faries D, Salkever D, Slade E, Peng X, et al. The cost of relapse and the predictors of relapse in the treatment of schizophrenia. BMC Psychiatry. 2010;10(1):2.

12. Ramon S, Healy B, Renouf N. Recovery from mental illness as an emergent concept and practice in Australia and the UK. Int J Soc Psychiatry. 2007;53(2):108-22.

13. Substance Abuse and Mental Health Services Administration, National Consensus Conference on Mental Health Recovery and Systems Transformation. Dept of Health and Human Services, Rockville, MD. 2005.

14. Heskestad S, Tytlandsvik M. [Patient-guided crisis admissions for severe psychotic conditions]. Tidsskr Nor Laegeforen. 2008;128(1):32-5.

15. Olso TM, Gudde CB, Moljord IE, Evensen GH, Antonsen DO, Eriksen L. More than just a bed: mental health service users' experiences of self-referral admission. Int J Ment Health Syst. 2016;10:11.

16. Strand $M$, von Hausswolff-Juhlin Y. Patient-controlled hospital admission in psychiatry: $A$ systematic review. Nord J Psychiatry. 2015;69(8):574-86.

17. Gudde $C B$, Ols $\varnothing \mathrm{TM}$, Antonsen $D \varnothing, \mathrm{R} \varnothing \mathrm{M}$, Eriksen $\mathrm{L}$, Vatne S. Experiences and preferences of users with major mental disorders regarding helpful care in situations of mental crisis. Scand J Public Health. 2013;41(2):185-90.

18. Organization WH. International statistical classification of diseases and health related problems (The) ICD-10: World Health Organization; 2004.

19. Lieberman JA, Perkins D, Belger A, Chakos M, Jarskog F, Boteva K, et al. The early stages of schizophrenia: speculations on pathogenesis, pathophysiology, and therapeutic approaches. Biol Psychiatry. 2001;50(11):884-97.

20. Lysaker PH, Buck KD. Is Recovery from schizophrenia possible? An overview of concepts, evidence, and clinical implications. Prim psychiatry. 2008;15(6):60-5.

21. Silverstein SM, Bellack AS. A scientific agenda for the concept of recovery as it applies to schizophrenia. Clin Psychol Rev. 2008;28(7):1108-24.

22. Schoeyen HK, Birkenaes AB, Vaaler AE, Auestad BH, Malt UF, Andreassen OA, et al. Bipolar disorder patients have similar levels of education but lower socio-economic status than the general population. J Affect Disord. 2011;129(1):68-74. 
23. Rise MB, Evensen GH, Moljord IE, Rø M, Bjørgen D, Eriksen L. How do patients with severe mental diagnosis cope in everyday life-a qualitative study comparing patients' experiences of selfreferral inpatient treatment with treatment as usual? BMC Health Serv Res. 2014;14(1):347.

24. First MB, Spitzer RL, Gibbon M, Williams JB. Structured Clinical Interview for DSM-IV-TR Axis I Disorders, Research Version, Patient Edition. (SCID-I/P). New York: Biometrics Research, New York State Psychiatric Institute; 2002.

25. Eaton WW, Thara R, Federman B, Melton B, Liang K-y. Structure and course of positive and negative symptoms in schizophrenia. Arch Gen Psychiatry. 1995;52(2):127-34.

26. Diamond RJ. Recovery from a psychiatrist's viewpoint. Postgrad Med. 2006:54-62. 


\section{Figure 1}

\section{Flow chart}

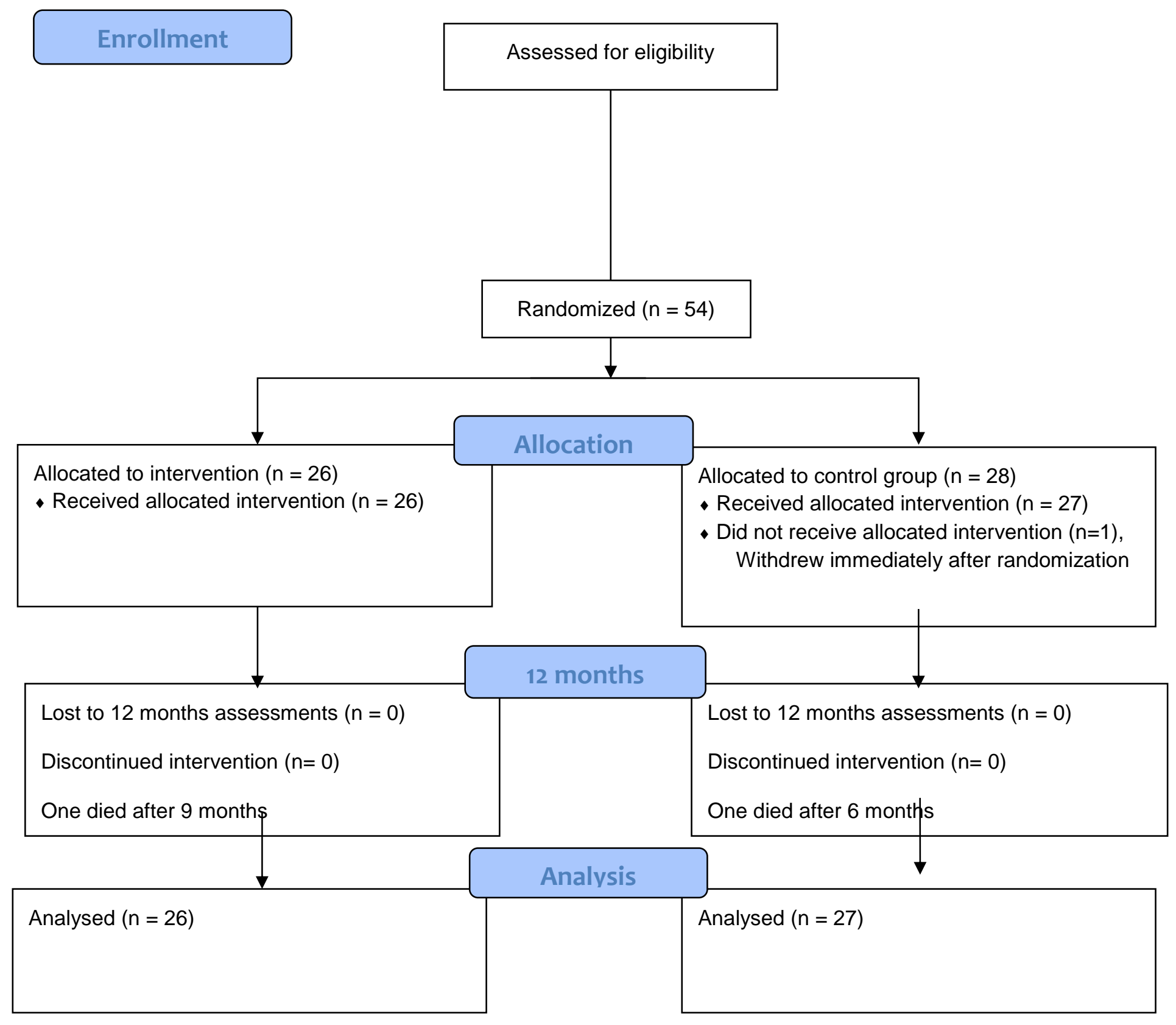


Figure 1:

Flow Chart 


\section{Table 1}

Demographic and clinical characteristics at baseline.

\begin{tabular}{|c|c|c|c|}
\hline & All & $\begin{array}{l}\text { Self-referral to } \\
\text { inpatient treatment }\end{array}$ & $\begin{array}{l}\text { Treatment } \\
\text { as usual }\end{array}$ \\
\hline Patients & $\mathrm{N}=53$ & $N=26$ & $\mathrm{~N}=27$ \\
\hline Age, mean (SD) & $40.3(13.0)$ & $45.7(12.5)$ & $35.2(11.6)$ \\
\hline Female No. & $22(42 \%)$ & $12(46 \%)$ & $10(37 \%)$ \\
\hline Days hospitalized 12 months preceding study entry & $72(31-220.5)$ & $118.5(26-230.5)$ & $53(29-157)$ \\
\hline Admissions 12 months preceding study entry & $2.87(1.86)$ & $3.19(2.14)$ & $2.56(1.53)$ \\
\hline $\begin{array}{l}\text { Days under coercion } 12 \text { months preceding study } \\
\text { entry }\end{array}$ & 81.77 (133.7) & $97.8(132.5)$ & $66.4(135.5)$ \\
\hline $\begin{array}{l}\text { Participants under coercion } 12 \text { months preceding } \\
\text { study entry }\end{array}$ & $20(38 \%)$ & $13(50 \%)$ & $7(26 \%)$ \\
\hline Diagnoses ICD 10 & & & \\
\hline Schizophrenia and other psychotic disorders & $40(75 \%)$ & $18(69 \%)$ & $22(81 \%)$ \\
\hline Bipolar disorder & $13(25 \%)$ & $8(31 \%)$ & $5(19 \%)$ \\
\hline Alcohol or drug abuse in history & $16(30 \%)$ & $8(31 \%)$ & $8(30 \%)$ \\
\hline
\end{tabular}


Table 2.

Number of inpatient days, admissions, outpatient contact and use of coercion 12 months

following study entry, given as median (interquartile 25-75) or numbers with percentage.

\begin{tabular}{|c|c|c|c|c|}
\hline & All & $\begin{array}{l}\text { Self-referral to } \\
\text { inpatient } \\
\text { treatment }\end{array}$ & $\begin{array}{l}\text { Treatment } \\
\text { as usual }\end{array}$ & $\mathrm{p}$ \\
\hline & $\mathrm{N}=53$ & $\mathrm{~N}=26$ & $\mathrm{~N}=27$ & \\
\hline \multicolumn{5}{|l|}{ Inpatient days } \\
\hline All\# & $34(11.5-94.5)$ & 39.5 (15.5-84.25) & $33(3-96)$ & $.33 *$ \\
\hline SRIT, CMHC, Hospital & $33(11.5-85)$ & 35.5 (15.5-70.25) & $33(3-96)$ & $.42 *$ \\
\hline CMHC including SRIT & $22(8-64.5)$ & $29.5(15.5-62.75)$ & $19(1-87)$ & $.23 *$ \\
\hline Acute ward at hospital & $0(0-7)$ & $0(0-8.25)$ & $0(0-6)$ & $.56^{*}$ \\
\hline $\begin{array}{l}\text { Acute and rehabilitation ward at } \\
\text { hospital }\end{array}$ & $0(0-7)$ & $0(0-9.25)$ & $0(0-6)$ & $.61 *$ \\
\hline Community services & $0(0-0)$ & $0(0-0)$ & & \\
\hline SRIT & & $5(0-17)$ & & \\
\hline \multicolumn{5}{|l|}{ Admissions } \\
\hline All\# & $3(1-6.5)$ & $4.5(1-8)$ & $2(1-5)$ & $.13 *$ \\
\hline SRIT, CMHC, Hospital & $3(1-6.5)$ & $4.5(1-8)$ & $2(1-5)$ & $.14^{*}$ \\
\hline CMHC including SRIT & $2(1-5)$ & $3(1-6)$ & $2(1-3)$ & $.02 *$ \\
\hline Acute ward at hospital & $0(0-2)$ & $0(0-1.3)$ & $0(0-2)$ & $.40 *$ \\
\hline SRIT & & $1.5(0-4.25)$ & & \\
\hline \multicolumn{5}{|l|}{ Outpatient consultations } \\
\hline All & $27(8.5-60)$ & $26.5(8.75-60.75)$ & $30(7-60)$ & $.89 *$ \\
\hline
\end{tabular}


Patients admitted

SRIT

SRIT, CMHC

Acute ward at hospital

$47(88.7 \%)$

$20(76.9 \%)$

Patients under coercion

$21(39.6 \%)$

$25(96.2 \%)$

$22(81.5 \%) \quad .10$

$18(34 \%)$

$9(35 \%)$

$12(44.4 \%) \quad .47$

Days under coercion

$0(0-103)$

$10(38 \%)$

$8(30 \%) \quad .50$

Analyzed with Mann-Whitney test* or Chi-Square test

SRIT: Self-referral to inpatient treatment

CMHC: Community Mental Health Center

\# All include admissions and inpatient days in hospital, SRIT, CMHC, and community services. 